\title{
The First Siphoviridae Family Bacteriophages Infecting Bordetella bronchiseptica Isolated from Environment
}

\author{
Aleksandra Petrovic $^{1} \cdot$ Rok Kostanjsek $^{2} \cdot$ Gabor Rakhely $^{3} \cdot$ Petar Knezevic $^{1}$ (D)
}

Received: 28 April 2016 / Accepted: 24 August 2016

(C) Springer Science+Business Media New York 2016

\begin{abstract}
Bordetella bronchiseptica is a well-known etiological agent of kennel cough in dogs and cats and one of the two causative agents of atrophic rhinitis, a serious swine disease. The aim of the study was to isolate $B$. bronchiseptica bacteriophages from environmental samples for the first time. A total of 29 phages from 65 water samples were isolated using the strain ATCC 10580 as a host. The lytic spectra of the phages were examined at 25 and $37^{\circ} \mathrm{C}$, using 12 strains of B. bronchiseptica. All phages were able to plaque on $25.0 \%$ to $41.7 \%$ of the strains. The selected phages showed similar morphology (Siphoviridae, morphotype B2), but variation of RFLP patterns and efficacy of plating on various strains. The partial genome sequence of phage vB_BbrS_CN1 showed its similarity to phages from genus Yuavirus. Using PCR, it was confirmed that the phages do not originate from the host strain, and environmental origin was additionally confirmed by the analysis of host genome sequence in silico and plating heated and unheated samples in parallel. Accordingly, this is the first isolation of $B$. bronchiseptica phages from environment and the first isolation and characterization of phages of B. bronchiseptica belonging to family Siphoviridae.
\end{abstract}

Electronic supplementary material The online version of this article (doi:10.1007/s00248-016-0847-0) contains supplementary material, which is available to authorized users.

Petar Knezevic

petar.knezevic@dbe.uns.ac.rs

1 Faculty of Sciences, Department of Biology and Ecology, University of Novi Sad, Trg Dositeja Obradovica 3, 21000 Novi Sad, Serbia

2 Biotechnical Faculty, Department of Biology, University of Ljubljana, Vecna pot 111, Ljubljana 1000, Slovenia

3 Department of Biotechnology, University of Szeged, Temesvari krt. 62, H-6726 Szeged, Hungary
Keywords Bordetella bronchiseptica $\cdot$ Bacteriophage · Isolation · Siphoviridae

\section{Introduction}

Bordetella bronchiseptica is a gram-negative coccobacillus, a microbita of upper parts of the respiratory tract of pigs, dogs, cats, rabbits, rats, horses, and other animals [1]. At the same time, it is a well-known etiological agent of various animal diseases, including infectious tracheobronchitis, conjuctivitis, rhinitis, mandibular lymphadenopathy, and pneumonia. In dogs and cats, it is one of the main causes of infective tracheobronchitis, known as kennel cough. In pigs, it is a causative agent of two diseases - bronchopneumonia and atrophic rhinitis. In the latter, $B$. bronchiseptica produces potent dermonecrotic toxin causing the atrophy of nasal septum and turbinate, as well as nasal and facial bones. This impairs swine feeding and consequently causes poor progression with great economic losses [2]. The disease has more severe consequences in mixed infections of $B$. bronchiseptica and Pasteurella multocida [3]. B. bronchiseptica is also a frequently detected microorganism in upper respiratory infections in rabbits, commonly called "snuffles" [4]. It can also be a pathogen of other animals, such as guinea pigs and horses $[5,6]$, but rarely of humans, with exception of immunocompromised individuals [7].

Apart from the bacteriophages of human pathogens, which have been studied widely, the phages of animal pathogens and opportunistic bacteria, including $B$. bronchiseptica, have mainly been neglected [8-10]. Although B. bronchiseptica was discovered more than a century ago [11], there is no reference describing its bacteriophages, except for a few related temperate bacteriophages induced from clinical strains, belonging to family Podoviridae $[12,13]$. B. bronchiseptica 
bacteriophage examination, however, is important from several aspects. For many multiple resistant bacteria, bacteriophages can be an alternative to conventional antibiotics [14], and $B$. bronchiseptica possesses innate or acquired antibiotic resistance to $\beta$-lactams, macrolides, tetracyclines, sulphonamides, and aminoglycosides, usually used for the treatment of animals with respiratory infections [15]. Furthermore, phage genomes can be new sources of genes for lytic enzymes, i.e., enzybiotics, as well as other valuable proteins [16]. The examination of $B$. bronchiseptica temperate phages can also explain potential prophage involvement in $B$. bronchiseptica virulence and the phenomenon of lysogenic conversion. Finally, their examination can also add to general knowledge of phage biology, as well as to their abundance and diversity in the environment.

The aim of this study was to isolate $B$. bronchiseptica-specific bacteriophages from environmental samples for the first time and to partially characterize them.

\section{Materials and Methods}

\section{Samples Origin}

A total of 65 samples from Serbia (59), Hungary (1), Georgia (1), Egypt (2), Turkey (1), and Switzerland (1) were collected during 2013 and 2014 including surface water from various origin (Table 1). Some of the municipal wastewater samples were taken repetitively from the same locations, but in different time intervals, so that all phages were obtained from geographically and/or chronologically different sources. The samples were taken aseptically and delivered to the laboratory in a short time. If this was not feasible, the samples were kept at $+4{ }^{\circ} \mathrm{C}$ for no longer than $48 \mathrm{~h}$.

\section{Bacterial strains}

Twelve strains of B. bronchiseptica were used in the study, including one reference strain (ATCC 10580), six strains of animal origin (973, L:594, BbChiot, CV1, LORD, and 3416), and five of human origin (R1, LEG, SEI, DEL, and S2). Besides $B$. bronchiseptica, 14 strains belonging to various taxa were used for phage lytic spectra determination: Alcaligenes faecalis (JR-22), Acinetobacter baumannii (ATCC BAA-747 and ATCC 19606), Plesiomonas shigeloides (ATCC 51903 and ATCC 14029), Escherichia coli (ATCC 13706), Staphylococcus aureus subsp. aureus (ATCC 11632), Eneterococcus faecalis (laboratory strain 36913 EXB-U53), Salmonella enterica subsp. enterica serovar Eneritidis (ATCC 13076), Klebsiella pneumoniae (laboratory strain 36913), Providencia rettgeri (ATCC 9250), Providencia stuartii (ATCC 49809), Morganella morganii subsp. morganii (ATCC 25829), and Proteus mirabilis (ATCC 35659). All bacteria were stocked in Luria-Bertani broth (LB) containing glycerol ( $v / v$ 10:1) at $-70{ }^{\circ} \mathrm{C}$. For the purpose of the experiments, they were cultivated overnight in $\mathrm{LB}$ at $37^{\circ} \mathrm{C}$.

\section{Phage Isolation}

B. bronchiseptica-specific phages were isolated using in parallel a single layer method with Luria-Bertani and Tryptone Salt Agar with ATCC 10580 strain as a phage host. All samples were prepared for isolation by filtration through a filter with pore diameter $0.45 \mu \mathrm{m}$, except two wastewater swine farm samples, which were filtered previously through filter paper because of visible impurities. Bacteriophage presence was determined in $100 \mathrm{ml}$ of sample according to U.S. EPA (2001) [17]. The plates were incubated $24-48 \mathrm{~h}$ at $37^{\circ} \mathrm{C}$ and

Table 1 Water samples and isolated phages

\begin{tabular}{|c|c|c|c|}
\hline Origin of samples & No. of samples & No. of isolated phages & Phage designation \\
\hline River & 12 & 4 & vB_BbrS_RV1; vB_BbrS_RV2; vB_BbrS_RV3; vB_BbrS_RV4 \\
\hline Lakes & 12 & 5 & $\begin{array}{l}\text { vB_BbrS_LK1; vB_BbrS_LK2; vB_BbrS_LK3; vB_BbrS_LK4 } \\
\text { vB_BbrS_LK5 }\end{array}$ \\
\hline Fish pond water & 6 & 5 & $\begin{array}{l}\text { vB_BbrS_FP1; vB_BbrS_FP2; vB_BbrS_FP3; vB_BbrS_FP4; } \\
\text { vB_BbrS_FP5 }\end{array}$ \\
\hline Swamp & 4 & 2 & vB_BbrS_SW1; vB_BbrS_SW2 \\
\hline Creek & 6 & 3 & vB_BbrS_CR1; vB_BbrS_CR2; vB_BbrS_CR3 \\
\hline Canal & 5 & 3 & vB_BbrS_CN1; vB_BbrS_CN2; vB_BbrS_CN3 \\
\hline Animal water trough & 12 & 2 & vB_BbrS_TR1; vB_BbrS_TR2 \\
\hline Wastewater swine farm & 2 & 0 & - \\
\hline Municipal wastewater & 6 & 5 & $\begin{array}{l}\text { vB_BbrS_MW1; vB_BbrS_MW2; vB_BbrS_MW3; vB_BbrS } \\
\text { _MW4; vB_BbrS_MW5 }\end{array}$ \\
\hline Total & 65 & 29 & \\
\hline
\end{tabular}


examined for plaques. Phage isolation assay for negative samples was repeated in order to confirm the negative results. From each plate with plaques, only one plaque was transferred into $\mathrm{SM}$ buffer $\left(50 \mathrm{mmol} \mathrm{L}^{-1}\right.$ Tris- $\mathrm{HCl}$ [pH 7.5], $100 \mathrm{mmol} \mathrm{L}^{-1} \mathrm{NaCl}, 8 \mathrm{mmol} \mathrm{L}^{-1} \mathrm{MgSO}_{4}, 0.01 \%$ w/v gelatin) to avoid isolation of clonally identical phages. The phage suspensions were subsequently 10 -fold serially diluted, and the plaques were re-isolated three times.

\section{Phage Propagation and Purification}

The phages were propagated on solid LB medium by doubleagar overlay method. The plates with semi-confluent plaques on B. bronchiseptica lawns were soaked with SM buffer overnight at $4{ }^{\circ} \mathrm{C}$, and the obtained phage suspensions were centrifuged to remove bacterial cells $\left(13,000 \times g 15 \mathrm{~min}\right.$ at $\left.4{ }^{\circ} \mathrm{C}\right)$, filtered (pore diameter 0.45 and $0.22 \mu \mathrm{m}$ ), and finally precipitated with $\mathrm{NaCl}$ and PEG8000. The concentrated phage suspensions were purified by ultracentrifugation in discontinuous $\mathrm{CsCl}$ density gradient [18]. The viral bands were collected and dialyzed overnight against SM buffer at $4{ }^{\circ} \mathrm{C}$. The plaque forming units per milliliter ( $\mathrm{PFU} \mathrm{ml}{ }^{-1}$ ) in the purified stocks were determined by preparation of serial 10-fold dilutions and double-agar overlay method in triplicate.

\section{Phage Host Range}

The analysis of phage lytic spectra was conducted using the spot method $\left(10 \mu \mathrm{l}\right.$ of $\left.10^{6} \mathrm{PFU} \mathrm{mL} \mathrm{m}^{-1}\right)$ on the lawns of potential hosts, with parallel incubation at 25 and $37^{\circ} \mathrm{C}$ for $24 \mathrm{~h}$. Apart from 12 B. bronchiseptica strains, the other above listed bacterial species were included in the experiment and incubated at $37^{\circ} \mathrm{C}$. After incubation, presence or absence of lysis was detected, and the obtained lytic zones were characterized as clear, clear with halo or turbid, and no reaction [9]. Each phage was tested against each bacterial strain in duplicate and in three independent experiments.

\section{Transmission Electron Microscopy}

Droplet method was applied for transmission electron microscopy of phages [19]. In brief, suspensions were transferred over copper grids with Formvar film and carbon, and the phages were adsorbed for $5 \mathrm{~min}$. In the next step, the excess of suspension was removed and fixed viral particles were contrasted with aqueous solution of uranyl acetate $(0.5-1 \%$ $w / v$ ) for $1 \mathrm{~min}$. After the samples were dried, viruses were observed using Philips CM 100 transmission electron microscope (Philips, Netherlands) at acceleration voltage of $80 \mathrm{kV}$. Electron micrographs of phages were obtained by a GatanBioscan CCD camera and Digital Micrograph software 3.4. (Gatan Inc., USA). The resulting images were used for phage morphology assessment. At least 10 virions were examined per phage isolate; the phage head and tail length and width were measured. Eight phages originating from various samples were examined by TEM: vB BbrS RV4, vB BbrS FP2, vB BbrS_CR1, vB_ B brS_LK3,

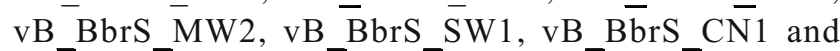
vB_BbrS_TR1. The obtained values were averaged and presented as mean $\pm \mathrm{SD}$.

\section{Efficiency of Plating}

The efficiency of plating was estimated using three $B$. bronchiseptica strains, lysed by all isolated phages at $37{ }^{\circ} \mathrm{C}$ : reference strain ATCC 10580 (the original host), 3416 and BbChiot (the alternative hosts). Series of 10-fold dilutions were applied to the lawn of bacteria, and the last dilution, which gave single plaques, was recorded. The titer obtained with alternative host was divided by the titer obtained with original host so that the results are expressed as relative values to the original host strain [20]. The experiment was carried out in duplicate in two independent occasions.

\section{One-Step Growth Curve}

One-step growth curve was performed as described by Nagayoshi et al. [21] with some modifications. An overnight culture was harvested by centrifugation $(5000 \mathrm{~g}, 5 \mathrm{~min})$ and resuspended in LB broth $\left(\sim 10^{5} \mathrm{CFU} / \mathrm{ml}\right)$. Phage vB_BbrS_CN1 was added to bacterial suspension at MOI $\sim 10$ and allowed to adsorb for $15 \mathrm{~min}$ with parallel incubation at 25 and $37^{\circ} \mathrm{C}$. After incubation, the mixture was washed with LB broth three times to remove any free virions $(5000 \mathrm{~g}, 5 \mathrm{~min})$. Thereafter, pelleted cells were resuspended in $100 \mathrm{ml}$ of LB broth, and incubation was continued at 25 and $37{ }^{\circ} \mathrm{C}$. Samples were taken, immediately centrifuged, and supernatant plated on LB agar to determine the phage titer at the beginning of the experiment and at 10-min intervals. Burst size, i.e., the number of released virions from cells, was determined as ratio of progeny phage to the initial number of infected cells. The experiment was performed in three independent occasions.

\section{Phage DNA Extraction and Restriction Enzyme Digestion}

The purified phage suspensions were treated with DNAse I and RNAse for $2 \mathrm{~h}$ at $37{ }^{\circ} \mathrm{C}$. Thereafter, DNAse was inactivated at $65{ }^{\circ} \mathrm{C}$ for $1 \mathrm{~h}$ and proteinase $\mathrm{K}\left(50 \mu \mathrm{g} \mathrm{ml}^{-1}\right)$, SDS $(0.5 \%)$, and EDTA $\left(20 \mathrm{mmol}^{-1}\right)$ were added to the phage suspension and incubated at $56^{\circ} \mathrm{C}$ for $1 \mathrm{~h}$ in order to release DNA from virions. A standard phenol-chloroform method was used to extract phage DNA [18]. The DNA pellet was washed with $70 \%$ ethanol, dried, and resuspended in TE overnight at $4{ }^{\circ} \mathrm{C}$.

The phage DNA was digested using nine FastDigest endonucleases (Thermo Fisher Scientific, Inc.) according to 
manufacturer recommendation: SmaI, HinfI, BamHI, XbaI, SalI, PvuI, BglII, EcoRV, and DraI. DNA fragments were visualized on agarose gels with ethidium bromide under UV light and documented by BioDoc system (Biometra, Germany).

\section{Partial Genome Sequencing}

The extracted and purified phage vB_Bbr_CN1 genomic DNA was partially sequenced by Ion Torrent method, with $50-150$-fold coverage. De novo assembly was carried out with CLC Genomics Workbench 6.5 and Mira 4. Open reading frame (ORF) prediction was done using GenemarkS, Glimmer, and Rast [22-24]. The obtained sequence was analyzed by BLASTn algorithm (Basic Local Alignment Search Tool) [25] and used to create primers for the experiment performed in order to confirm the phage environmental origin.

\section{Confirmation of Phage Environmental Origin}

To confirm that the isolated phages originated from the samples and were not induced from bacterial host ATCC 10580, the presence of prophages in the host genome sequence (NZ_CM002881.1) was examined using the PHAST server (phast.wishartlab.com/). PHAST (PHAge Search Tool) is designed to identify, annotate, and graphically display prophage sequences within bacterial genomes [26]. The identified phage genome was subjected to theoretical digestion with endonucleases (http://tools.neb. com/REBsites/index.php) [27] and compared to obtained RFLP patterns of isolated phages. In addition, selected samples were heated at $100{ }^{\circ} \mathrm{C}$ for $20 \mathrm{~min}$ and plated in parallel with unheated samples to confirm the phage environmental origin. Finally, based on the partial genome sequence of vB_BbrS_CN1, a primer pair was created with expected product of $241 \mathrm{bp}$ (Bbr-F 5' TGACTTCATGGTTG CCGTTC $3^{\prime}$ and Bbr-R 5' TCGGGAGCGTGATTTCAGTA $3^{\prime}$ ) and a PCR with host genomic DNA extracted using GeneJET Genomic DNA Purification Kit (Fermentas, Lithuania) was performed. A primer pair for housekeeping gene recA of $B$. bronchiseptica was designed (Bbr_recA-F: 5' ATGGCGACAACGAGGTCGAA 3' and Bbr_recA-R: 5' CAGCAGGTCGGTCAGGTTGA $3^{\prime}$ ) and applied as a control of bacterial DNA quality, with the expected product of $263 \mathrm{bp}$. Thermal cycling conditions were as follows: an initial cycle of $94^{\circ} \mathrm{C}$ for $5 \mathrm{~min}$ followed by 35 cycles of $94^{\circ} \mathrm{C}$ for 30 $\mathrm{s}$, annealing at $55^{\circ} \mathrm{C}$ for $20 \mathrm{~s}$, and extension at $72{ }^{\circ} \mathrm{C}$ for $60 \mathrm{~s}$, with a final 7 -min extension at $72{ }^{\circ} \mathrm{C}$. PCR products were analyzed on $2 \%$ agarose gel with ethidium bromide. A positive control was phage DNA and a negative distilled water. In addition, DNAs of other isolated phages were used as templates, in order to confirm the presence of target genes in phage genomes, i.e., to examine their similarity.

\section{Results}

In this study, B. bronchiseptica phages were isolated for the first time from environmental samples. The phages were successfully isolated from $44.6 \%$ samples (29 out of 65 ) (Table 1). The most successful phage isolation was from municipal wastewaters, since all samples were positive, while no single phage was isolated from swine farm wastewater. From all other types of samples, B. bronchiseptica phages were isolated, but with various success. In some samples, the number of bacteriophages was relatively high (more than $10 \mathrm{PFU} /$ $\mathrm{ml}$; the results are not shown).

From each positive sample, one plaque was isolated and 29 phages were further examined to determine their lytic spectra. On the original host lawn, all phages formed clear plaques, with diameter in range $0.5-2 \mathrm{~mm}$, while on the lawn of alternative hosts the plaques were turbid (Table 2). Among 12 $B$. bronchiseptica strains, 5 strains were sensitive to bacteriophages including the original host, while 7 strains were not lysed by any phage. The lytic spectra of phages at 25 and $37{ }^{\circ} \mathrm{C}$ significantly differed, and besides the original strain, the phages were able to lyse 2 or 4 strains in addition. Accordingly, the phages possessed narrow or moderate activity spectra. Based on the host range, it was possible to observe only three different lytic groups at $37^{\circ} \mathrm{C}$ : first group was able to lyse $25 \%$ (16 phage isolates), while the phages of second and third group were able to lyse $33.3 \%$ of the strains (second group comprises 11 and third group comprises 2 phage isolates). The results of lytic spectra obtained at $25^{\circ} \mathrm{C}$ were more diverse than at $37^{\circ} \mathrm{C}$. In effect, six groups were differentiated: the first three groups were able to lyse $33.3 \%$ of the strains (13 phage isolates in first, two in second, and one in third group), the phages of the fourth group were able to lyse $41.7 \%$ (11 phage isolates) and the fifth and the sixth group were able to lyse $25 \%$ of B. bronchiseptica strains (one phage isolate in each group).

Efficiency of plating (EOP) of B. bronchiseptica bacteriophages is shown in Table 3. According to the results, six phages showed higher EOP (10 and 100) on non-original host strains than on the original ATCC 10580. A large number of phages show equal EOP (1) for original and two alternative hosts. As expected, the most phages showed lower EOP in comparison to the original host, ranging from 0.001 to 0.1 . One-step growth curve showed long latent period at both 25 and $37{ }^{\circ} \mathrm{C}$ (90 and $110 \mathrm{~min}$, respectively), and more than double higher burst size at $37^{\circ} \mathrm{C}$ (approx. 6 vs. 13) (Fig. 1).

Morphological characteristics of eight phages were examined, and since all these phages had highly similar morphology, only morphological details of four phages selected based on different RFLP and/or lytic activity are presented in Fig. 2 and Table 4. Based on their morphological properties, all phages appeared to be relatively uniform: they belong to order Caudovirales, as they have binary structure consisting of head 


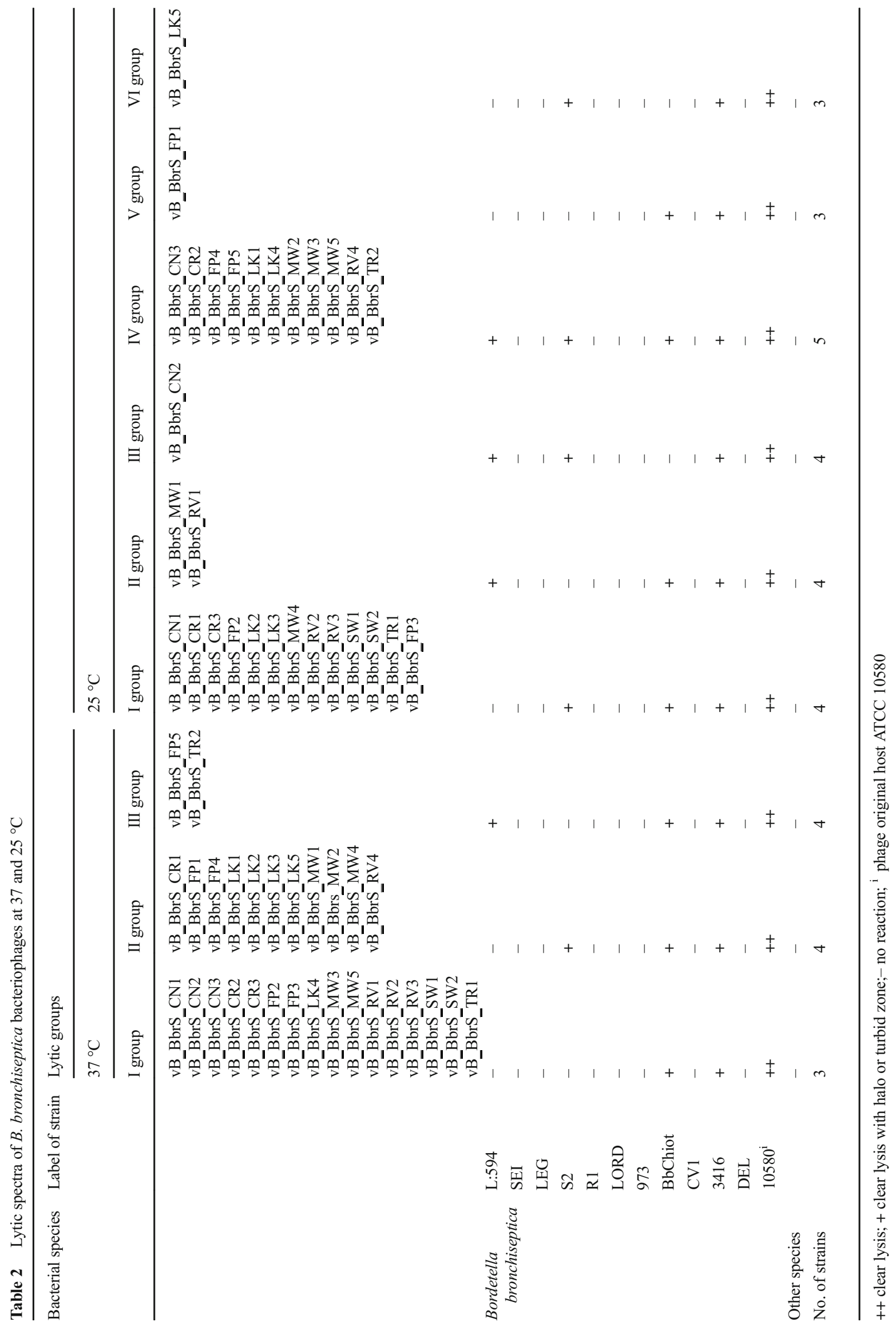


Table 3 Efficiency of plating (EOP) of B. bronchiseptica bacteriophages on two alternative hosts

\begin{tabular}{|c|c|c|c|c|c|c|}
\hline \multirow{2}{*}{$\begin{array}{l}\text { B. bronchiseptica } \\
\text { alternative host strains }\end{array}$} & \multicolumn{6}{|l|}{ EOP } \\
\hline & 100 & 10 & 1 & 0.1 & 0.01 & 0.001 \\
\hline BbChiot & vB_BbrS_LK4 & $\begin{array}{l}\text { vB_BbrS_CN1 } \\
\text { vB_BbrS_CR3 } \\
\text { vB_BbrS_LK5 } \\
\text { vB_BbrS_MW3 }\end{array}$ & $\begin{array}{l}\text { vB_BbrS_CN2 } \\
\text { vB_BbrS_CN3 } \\
\text { vB_BbrS_FP1 } \\
\text { vB_BbrS_FP3 } \\
\text { vB_BbrS_LK2 } \\
\text { vB_BbrS_MW2 } \\
\text { vB_BbrS_RV1 } \\
\text { vB_BbrS_RV4 } \\
\text { vB_BbrS_SW2 } \\
\text { vB_BbrS_TR1 } \\
\text { vB_BbrS_TR2 } \\
\text { vB_BbrS_MW5 }\end{array}$ & $\begin{array}{l}\text { vB_BbrS_CR2 } \\
\text { vB_BbrS_FP5 } \\
\text { vB_BbrS_LK1 } \\
\text { vB_BbrS_LK3 } \\
\text { vB_BbrS_MW1 } \\
\text { vB_BbrS_MW4 } \\
\text { vB_BbrS_RV3 } \\
\text { vB_BbrS_SW1 }\end{array}$ & $\begin{array}{l}\text { vB_BbrS_CR1 } \\
\text { vB_BbrS_FP4 } \\
\text { vB_BbrS_RV2 }\end{array}$ & vB_BbrS_FP2 \\
\hline 3416 & - & $\begin{array}{l}\text { vB_BbrS_FP1 } \\
\text { vB_BbrS_LK5 }\end{array}$ & $\begin{array}{l}\text { vB_BbrS_CN3 } \\
\text { vB BbrS_CR3 } \\
\text { vB_BbrS_FP2 } \\
\text { vB_BbrS_LK4 } \\
\text { vB_BbrS_RV1 } \\
\text { vB_BbrS_TR1 } \\
\text { vB_BbrS_TR2 }\end{array}$ & $\begin{array}{l}\text { vB_BbrS_CN2 } \\
\text { vB_BbrS_CR2 } \\
\text { vB_BbrS_FP3 } \\
\text { vB_BbrS_FP5 } \\
\text { vB_BbrS_LK1 } \\
\text { vB_BbrS_LK2 } \\
\text { vB_BbrS_MW1 } \\
\text { vB_BbrS_MW2 } \\
\text { vB_BbrS_MW3 } \\
\text { vB_BbrS_MW4 } \\
\text { vB_BbrS_MW5 } \\
\text { vB_BbrS_RV4 }\end{array}$ & $\begin{array}{l}\text { vB_BbrS_CN1 } \\
\text { vB_BbrS_CR1 } \\
\text { vB_BbrS_FP4 } \\
\text { vB_BbrS_LK3 } \\
\text { vB_BbrS_RV2 } \\
\text { vB_BbrS_RV3 } \\
\text { vB_BbrS_SW1 } \\
\text { vB_BbrS_SW2 }\end{array}$ & - \\
\hline
\end{tabular}

and tail [28, 29]. The phages have head of length approx. $72 \mathrm{~nm}$ and the width $52 \mathrm{~nm}$. They possessed a long, noncontractile, and flexible tail of length approx. $160 \mathrm{~nm}$ and the width $12 \mathrm{~nm}$. Based on all characteristics mentioned above, $B$. bronchiseptica-specific phages belong to the family Siphoviridae and possess the prolate head, corresponding to Bradley's group B2 [30].

The nucleic acids of selected phages were extracted and digested using nine restriction enzymes. The digestion of

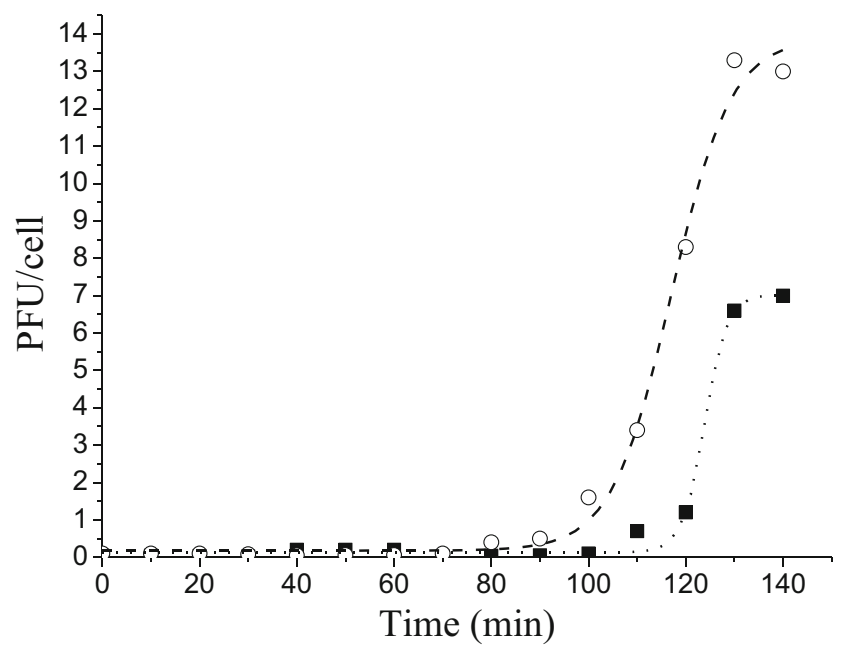

Fig. 1 One-step growth curve of phage vB_BbrS_CN1 at $25^{\circ} \mathrm{C}$ (square) and $37^{\circ} \mathrm{C}$ (circle)

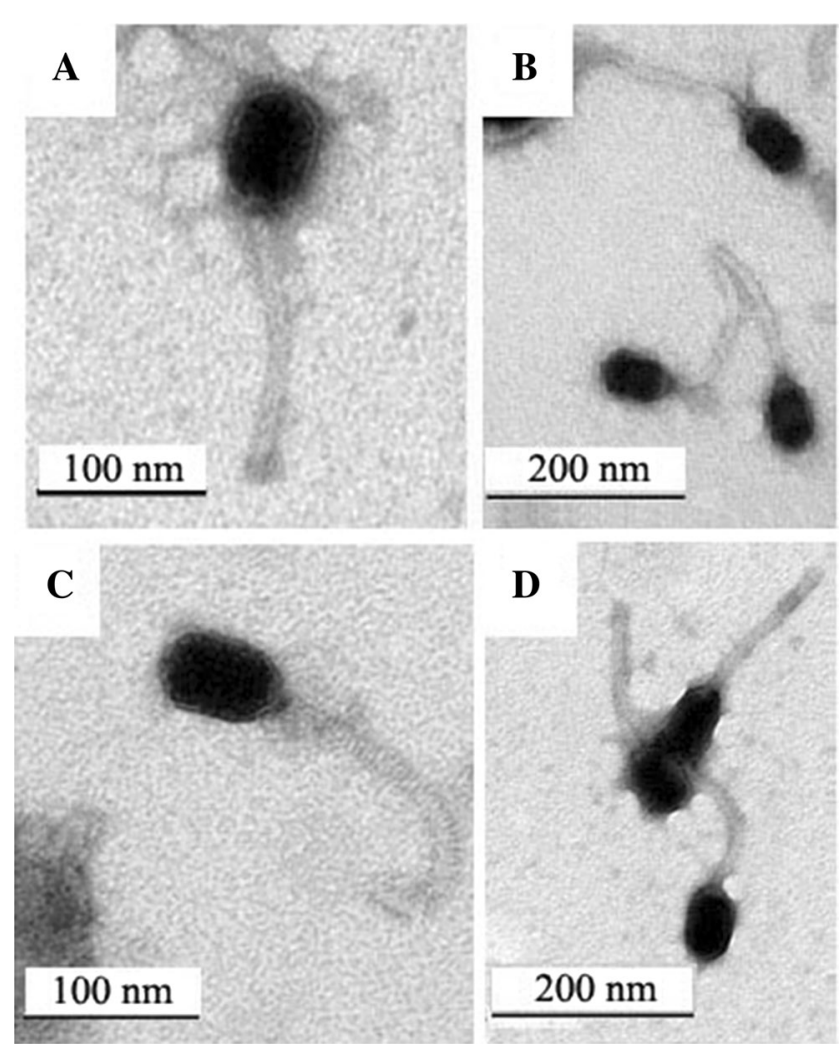

Fig. 2 Electron micrographs of selected $B$. bronchiseptica phages: a vB BbrS_LK3, b vB_BbrS_FP2, c vB_BbrS_RV4, d vB_Bbr_CN1 
Table 4 Morphological characteristics of phages

\begin{tabular}{lllll}
\hline Phage & \multicolumn{4}{l}{ Phage particle dimensions (nm) } \\
\cline { 2 - 5 } & Head lenght & Head width & Tail lenght & Tail width \\
\hline vB_BbrS_RV4 & $72.2 \pm 1.5$ & $50.6 \pm 2.2$ & $162.4 \pm 3.9$ & $11.8 \pm 1.6$ \\
vB_BbrS_FP2 & $70.5 \pm 3.5$ & $49.4 \pm 2.5$ & $158.9 \pm 1.9$ & $12.6 \pm 1.1$ \\
vB_BbrS_LK3 & $73.7 \pm 2.6$ & $54.9 \pm 1.8$ & $161.8 \pm 4.9$ & $13.1 \pm 1.3$ \\
vB_BbrS_CN1 & $72.1 \pm 1.3$ & $53.4 \pm 2.6$ & $160.8 \pm 3.1$ & $12.8 \pm 1.6$ \\
\hline
\end{tabular}

phage DNAs with BglII, PvuI, SalI, BamHI, XbaI, and DraI did not result in any visible fragment, due to the absence of restriction site or to the presence of modified bases. However, DNAs of all studied phages were cut by HinfI and SmaI. Both restriction endonucleases generated a great number of fragments (Fig. 3a, b), indicating the presence of many specific sites for these enzymes in phage DNAs. EcoRV gave patterns that allow genome size determination in phages vB_BbrS_CN1 and vB_BbrS_LK3 (Fig. 3c), which is estimated to be approx. $55 \mathrm{kbp}$. Using this enzyme, a faint band indicating a pac site was observed (Fig. 3c, arrow head).

By the genome examination of the strain ATCC 10580 by the PHAST server, only one phage was detected, highly related to a Cronobacter phage phiES15. However, the phage showed no similarity with any known phage when BLASTn was used. Its DNA is sensitive to all restriction endonucleases used in the study when theoretically digested, except for DraI and XbaI. Among enzymes used in the study, BamHI, DraI, and BglII are not sensitive to nucleotide methylation. Since BglII cannot cut DNA of any phage isolated in the study but can cut prophage DNA in the reference $B$. bronchiseptica strain in silico, it is clear that the isolated phages do not originate from the host strain. In contrast to unheated samples, no phage was isolated from heated samples, additionally confirming their origin from environmental samples and not from the bacterial host (Fig. 4).

The partial sequence of $2113 \mathrm{bp}$ of phage vB BbrS CN1 (Access. No. KX015888; supplementary file 1) showed $40 \%$ similarity with $P$. aeruginosa siphovirus phage LK04 (the result is not shown) belonging to genus Yuavirus when BLASTn was used. The PCR results with primer pair designed on the basis of vB_BbrS_CN1 sequence were negative since there were not annealing sites for the primers in total DNA of host strain (Fig. 5). The PCR was performed with DNAs of other selected phages and the product of the expected size was obtained.

\section{Discussion}

Bacteriophages are considered the most abundant entity in biosphere, whose estimated number is approx. $10^{30}$ with about $10^{8}$ species [31-33]. Up to date, many phages have been isolated, described, and their genomes have been sequenced. There are several reports on $B$. bronchiseptica phage isolation, but all of them were obtained from bacterial cultures by induction or spontaneous releasing, not from environmental samples. Rauch and Piccket [34] isolated 38 B. bronchiseptica specific phages from 48 strains and obtained three more as spontaneous virulent mutants of the isolates. Unfortunately, except from phage lytic spectra, other characteristics were not further examined. A temperate phage has also been noticed in a strain used for vaccine production [35]. The existence of this phage was only documented by
Fig. 3 RFLP profile of selected B. bronchiseptica phages DNAs digested with HinfI (a), SmaI (b), and EcoRV (c). M1: $1 \mathrm{kbp}$ marker; $M 2$ : 100 bp marker; M3: Lambda DNA/HindIII marker
A

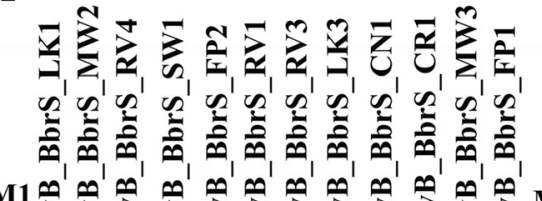

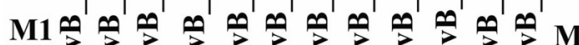

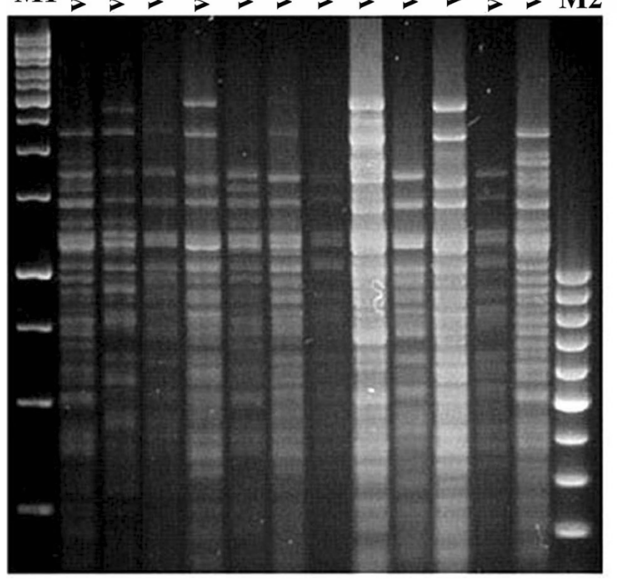

B
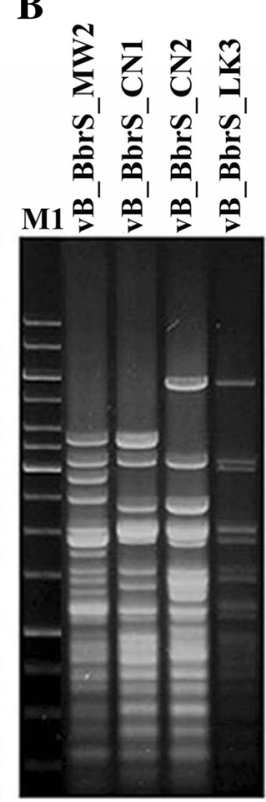

C

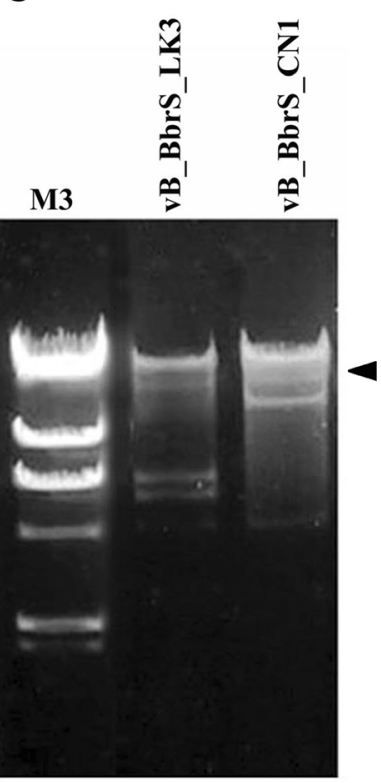


Fig. 4 Isolation of bacteriophages from a sample heated at $100{ }^{\circ} \mathrm{C}$ for $20 \mathrm{~min}$ (a) and the same unheated sample (b)
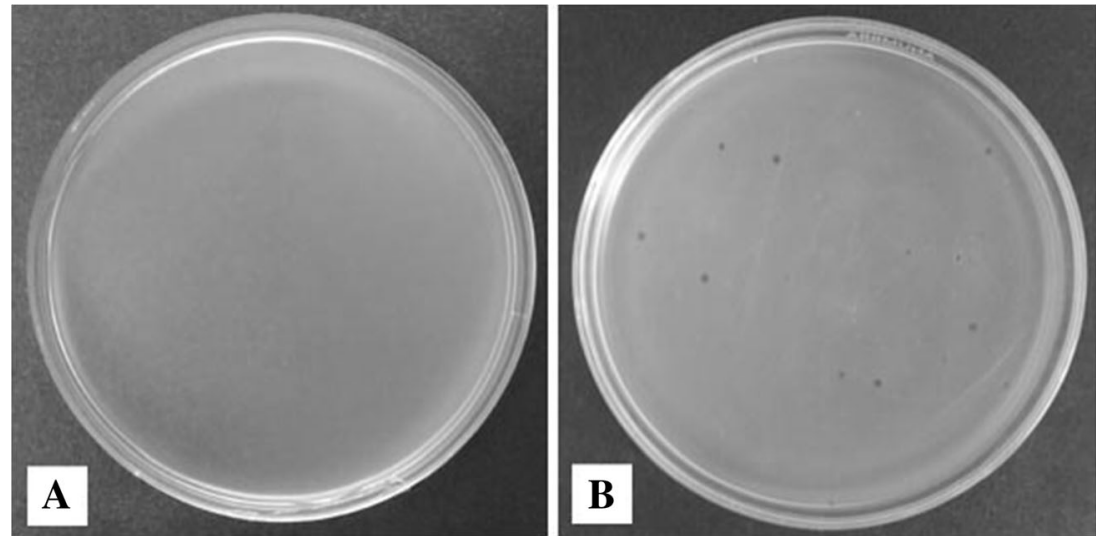

TEM, and according to a micrograph, it seems that it belongs to family Siphoviridae, morphotype B1. So far, only three highly related temperate Podoviridae (BPP-1, and its topic variants BMP-1 and BIP-1), identified in clinical isolates of B. bronchiseptica, have been described in more details and their genomes have been sequenced $[12,13]$.

For the first time, we isolated B. bronchiseptica bacteriophages from environment. The presence of $B$. bronchiseptica phages in water environment is very surprising, since phages are expected to inhabit environments in which their hosts exist [36], and $B$. bronchiseptica is not recognized as a water-related microorganism. It is also worth noticing that an enrichment procedure was not applied, but in some samples, the number of bacteriophages was relatively high (more than $10 \mathrm{PFU} / \mathrm{ml}$ ). Although $B$. bronchiseptica is associated with swine, the phage isolation was unsuccessful from samples taken from swine water troughs and swine wastewater. In this regard, it must be taken into account that the samples from swine farm were filtered through a paper filter, and this pretreatment can cause a phage loss [9]. However, it is also possible that the phages were absent in the samples or that their number was very low.
The results of phage lytic spectra at 25 and $37{ }^{\circ} \mathrm{C}$ significantly differed. The obtained differences are not surprising, since the bacterium possesses BvgAS two-component signal transduction system, which regulates expression of genes responsible for virulence and motility. Growing the bacteria in rich media at $37{ }^{\circ} \mathrm{C}$ will induce $\mathrm{Bvg}^{+}$phase for which the expression of virulence genes such as filamentous hemagglutinin (FHA), fimbriae, and bifunctional adenylatecyclase/ hemolysin (ACY) is typical. In the presence of millimolar concentration of modulators (nicotinic acid or $\mathrm{MgSO}_{4}$ ) or at temperatures below $30{ }^{\circ} \mathrm{C}, \mathrm{BvgAS}$ is negatively regulated (Bvg ${ }^{-}$phase) and the bacterium exhibits decreased virulence, with upregulated flagellin expression $[37,38]$. There is also a $\mathrm{Bvg}^{\mathrm{i}}$ phase, i.e., interphase with upregulated FHA and fimbriae, and downregulated ACY, which is considered responsible for biofilm formation [38, 39]. The B. bronchiseptica phenotypic variation caused by temperature change, particularly in strains S2 and L: 594, probably had an impact on phage receptor expression as these two strains were more susceptible to a phage infection at $25^{\circ} \mathrm{C}$. It should also be stressed that $\mathrm{Bvg}^{\mathrm{i}}$ and $\mathrm{Bvg}^{-}$phases are temperature dependent and thus expected to be dominant in environmental and not in clinical samples. The broader phage activity spectra at $25^{\circ} \mathrm{C}$ are in

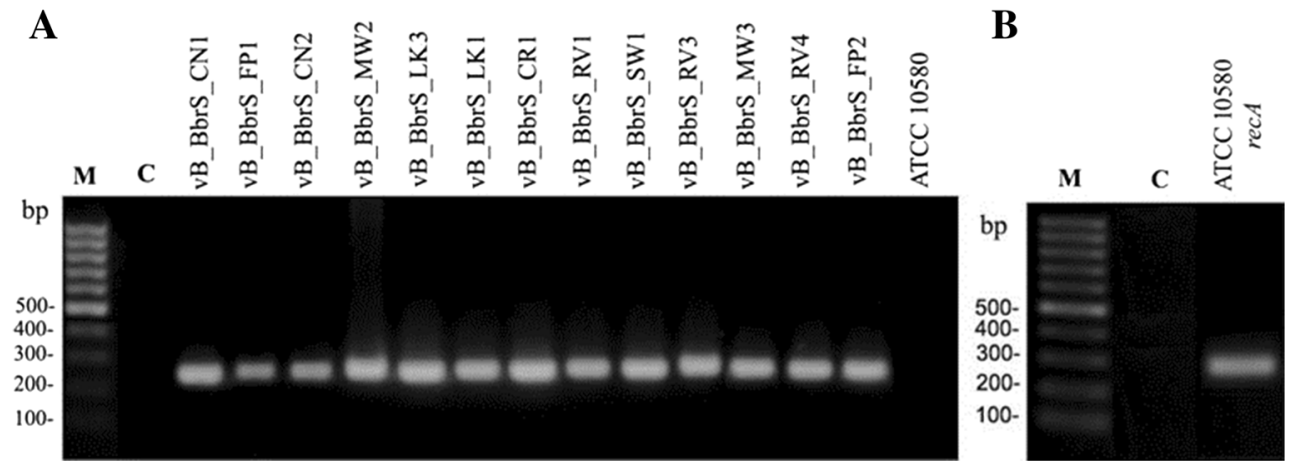

Fig. 5 PCR products: a obtained with primer pair Bbr-F and Bbr-R, created based on $\mathrm{vB}$ BbrS CN1 partial DNA sequence. All phages gave the expected product of $241 \mathrm{bp}$, while the product is absent when host
DNA was used as a template; $\mathbf{b}$ positive control of bacterial DNA quality with primer pair Bbr-recA. $M$ : $100 \mathrm{bp}$ marker; $C$ : a negative control with distilled water 
accordance with their environmental origin. However, other reasons for the temperature difference in lytic spectra should not be neglected, including the possibility that the function of some phage proteins could be affected by temperature (receptor ligands, repressor protein, etc.). One-step growth curve pointed to the long latent period and generally small burst size at both temperatures, indicating that optimal growth conditions for B. bronchiseptica were not achieved and/or that phages predominantly formed lysogens, rather than entering a lytic cycle.

Considering the phage relative abundance in the environmental samples, it was expected that the phages were not specific for B. bronchiseptica. Surprisingly, inclusion of the other species in lytic spectra determination showed that the phages were not able to lyse any of the examined species, including members of classes Betaproteobacteria (family Alcaligenaceae) and Gammaproteobacteria (families Enterobacteriaceae and Moraxelaceae) (Table 2). These results indicate the specificity of the phages, at least for the genus Bordetella, although the possibility that they infect other species or strains, particularly of enterobacteria not included here into lytic spectra examination should not be abandoned.

The obtained EOP results clearly indicate that for some phages, alternative B. bronchiseptica hosts were more appropriate for infection. The reason for this can be the presence of more phage receptors on bacterial cells that enhanced adsorption and subsequent infection. However, the presence of a contaminating temperate phage spontaneously released or induced from propagation strain in the purified lysates or prophage induction from the strains used for assay after infection with examined B. bronchiseptica phages cannot be excluded.

Restriction digestion of extracted nucleic acid revealed that all bacteriophages possessed dsDNA within the virions. Taking into account the results of RFLP profiles, it can be noted that the phages were very similar, although some of them showed considerable variations in patterns when HinfI and SmaI enzymes were used. Additionally, as the product of the expected size was obtained in all examined phages by the PCR method with primers created based on vB_BbrS_CN1 partial sequence, it is obvious that the phage genomes are highly related. Using EcoRV, it was possible to determine genome size of vB_BbrS_LK3 and vB_BbrS_CN1, which is

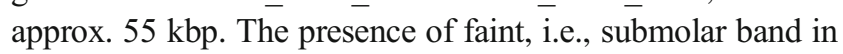
RFLP pattern, here obtained with EcoRV, is considered to be diagnostic of headfull packaging [40].

According to the PHAST server and in silico digestion by restriction endonuclease, the isolated phages in the study are distinctive from the prophage existing in $B$. bronchiseptica ATCC 10580 and are newly isolated. It is interesting to note that the phages are morphologicaly similar to those belonging to Yuavirus. According to the similarity of the phage vB_BbrS_CN1 sequence and morphology to member of this genus, there is a possibility that phages are able to form lysogens and thus inappropriate for application as an antimicrobial agent as whole virion. However, its lysine could be of interest for preparation of enzybiotics, so the phage genome should be further examined to detect the gene for endolysin.

The PCR with primer pair designed on the basis of vB_BbrS_CN1 sequence confirmed that phage has environmental origin since in total DNA of host strain there were not annealing sites for the primers. Additionally, phage environment origin was confirmed since in contrast to unheated samples, no phage was isolated from heated samples.

In summary, this is the first isolation of $B$. bronchiseptica bacteriophages from environmental samples in which they have proven to be relatively frequent and abundant. This is also the first time that the phages from family Siphoviridae specific for $B$. bronchiseptica are described in more details. All examined phages were highly related, showing similar morphology, but with certain variation in lytic spectra, EOP and RFLP patterns. Based on vB_BbrS_CN1 similarity to Yuavirus members that possess integrase, the phage is probably not useful for application as antimicrobial agent, but the future examination should be directed toward their endolysin characterization. More important, genome sequence and phage ability to form stable lysogeny can reveal its possible involvement in bacterial virulence, i.e., in the phenomenon of lysogenic conversion of their hosts.

Acknowledgments This work was supported by the project of Ministry of Education, Science and Technological Development of the Republic of Serbia, TR 31084 and Hungary-Serbia IPA Cross-border Co-operation Programme, project NNAA HUSRB/1203/214/250. The authors acknowledge Dr Nicole Guiso from Institut Pasteur, Paris, France and Mr. Aleksandra Markovic from Veterinary Institute Subotica, Serbia for providing $B$. bronchiseptica strains with their kindness and support. We thank Dr. Liljana Knezevic, University of Novi Sad Faculty of Sciences, for editing English language.

\section{References}

1. Yacoub AT, Katayama M, Tran J, Zadikany R, Kandula M, Greene $\mathrm{J}$ (2014) Bordetella bronchiseptica in immunosuppressed population - a case series and review. Mediterr J Hematol Infect Dis 6(1): e2014031. doi:10.4084/MJHID.2014.031

2. Brockmeier SL, Register KB, Magyar T, Lax AJ, Pullinger GD, Kunkle RA (2002) Role of dermonecrotic toxin of Bordetella bronchiseptica in the pathogenesis of respiratory disease in swine. Infect Immun 70(2):481-490. doi:10.1128/IAI.70.2.481-490.2002

3. Rutter JM (1985) Atrophic rhinitis in swine. Adv Vet Sci Comp Med 29:239-279

4. Burns EH Jr, Norman JM, Hatcher MD, Bemis DA (1993) Fimbriae and determination of host species specificity of Bordetella bronchiseptica. J Clin Microbiol 31(7):1838-1844

5. Egberink H, Addie D, Belák S, Boucraut-Baralon C, Frymus T, Gruffydd-Jones T, Hartmann K, Hosie MJ, Lloret A, Lutz H, Marsilio F, Pennisi MG, Radford AD, Thiryn E, Truyen U, Horzinek MK (2009) Bordetella bronchiseptica infeftion in cats. $\mathrm{ABCD}$ guidelines on prevention and management. J Feline Med Surg 11(7):610-614. doi:10.1016/j.jfms.2009.05.010 
6. Bradley A, Kinyon J, Frana T, Bolte D, Hyatt DR, Lappin MR (2012) Efficacy of intranasal administration of a modified live feline herpesvirus 1 and feline calicivirus vaccine against disease caused by Bordetella bronchiseptica after experimental challenge. J Vet Intern Med 26(5):1121-1125. doi:10.1111/j.19391676.2012.00982.x

7. Woolfrey BF, Moody JA (1991) Human infections associated with Bordetella bronchiseptica. Clin Microbiol Rev 4:243-255

8. Sulakvelidze A, Alavidze Y, Morris JG (2001) Bacteriophage therapy. Antimicrob Agents Chemother 3:649-659. doi:10.1128 /AAC.45.3.649-659.2001

9. Knezevic P, Kostanjsek R, Obreht D, Petrovic O (2009) Isolation of Pseudomonas aeruginosa specific phages with broad activity spectra. Curr Microbiol 59:173-180. doi:10.1007/s00284-009-9417-8

10. Chan BK, Abedon ST, Loc-Carrillo C (2013) Phage cocktails and the future of phage therapy. Future Microbiol 8(6):769-783. doi:10.2217/fmb.13.47

11. Ferry NS (1912) Bacillus bronchisepticus (bronchicanis): the cause of distemper in dogs and a similar disease in other animals. Vet J 68: 376-391

12. Liu M, Deora R, Doulatov SR, Gingery M, Eiserling FA, Preston A, Maskell DJ, Simons RW, Cotter PA, Parhill J, Miller JF (2002) Reverse transcriptase-mediated tropism switching in Bordetella bacteriophage. Science 295(5562):2091-20194. doi:10.1126 /science.1067467

13. Liu M, Gingery M, Doulatov SR, Liu Y, Hodes A, Baker S, Davis P, Simmonds M, Churcher C, Mungall K, Quail MA, Preston A, Harvill ET, Maskell DJ, Eiserling EA, Parkhill J, Miller JF (2004) Genomic and genetic analysis of Bordetella bronchiseptica encoding reverse transcriptase-mediated tropism-switching cassettes. J Bacteriol 186(5):1503-1517. doi:10.1128/JB.186.5.15031517.2004

14. Sulakvelidze A, Kutter E (2005) Bacteriophage therapy in humans. In: Kutter E, Sulakvelidze A (eds) Bacteriophages - biology and applications. CRC Press, Boca Raton, pp 381-436

15. Pruller S, Rensch U, Meemken D, Kaspar H, Kopp PA, Klein G, Kehrenberg C (2015) Antimicrobial susceptibility of Bordetella bronchiseptica isolates from swine and companion animals and detection of resistance genes. PLoS ONE 10(8):e0135703. doi:10.1371/journal.pone. 0135703

16. Drulis-Kawa Z, Majkowska-Skrobek G, Maciejewska B (2015) Bacteriophages and phage-derived proteins - application approaches. Curr Med Chem 22(14):1757-1773

17. USEPA (2001) Method 1602: detection of Male-specific $(\mathrm{F}+)$ and somatic coliphage in water by Single Agar Layer (SAL) procedure. EPA 821-R-01-029. Office of Water, Engineering and Analysis Division, Washington, DC

18. Sambrook J, Russell DW (2001) Molecular cloning: a laboratory manual, 3rd edn. Cold Spring Harbor Laboratory Press, New York

19. Bozzola JJ, Russell LD (1998) Electron microscopy—principles and techniques for biology, 2nd edn. Jones and Bartlett Publishers, Sundbury, p 670

20. Kutter E (2009) Phage host range and efficiency of plating, Bacteriophages, Methods and Protocols, vol 1. Isolation, Characterization and Interactions, pp. 141-149

21. Nagayoshi Y, Kumagae K, Mori K, Tashiro K, Nakamura A, Fujino Y, Yasuaki H, Iwamoto T, Kuhara S, Ohshima T, Katsumi T (2016) Physiological properties and genome structure of the hyperthermophilic filamentous phage $\varphi \mathrm{OH} 3$ which infects Thermus termophilus HB8. Front Microbiol 7:50. doi:10.3389 /fmicb.2016.00050

22. Besemer J, Lomdsadze A, Borodovsky M (2001) GeneMarkS: a self-training method for prediction of gene starts in microbial genomes. Implications for finding sequence motifs in regulatory regions. Nucleic Acids Res 29:2607-2618. doi:10.1093 /nar/29.12.2607

23. Delcher AL, Harmon D, Kasif S, White O, Salzberg SL (1999) Improved microbial gene identification with GLIMMER. Nucleic Acids Res 27(23):4636-4641. doi:10.1093/nar/27.23.4636

24. Aziz RA, Bartels D, Best AA, DeJongh M, Disz T, Edwards RA, Formsma K, Gerdes S, Elizabeth MG, Kubal M, Meyer F, Gary JO, Olson R, Osterman AL, Overbeek RA, McNeil LK, Paarmann D, Paczian T, Parrello B, Pusch GD, Reich C, Stevens R, Vassieva O, Vonstein V, Wilke A, Zagnitko O (2008) The RAST server: rapid annotation using subsystems technology. BMC Genomics 9:75. doi:10.1186/1471-2164-9-75

25. Altschul SF, Gish W, Miller W, Myers EW, Lipman DJ (1990) Basic local alignment search tool. J Mol Biol 215(3):403-410. doi:10.1016/S0022-2836(05)80360-2

26. Zhou Y, Liang Y, Lunch K, Dennis JJ, Wishart DS (2011) PHAST: a fast phage search tool. Nucleic Acids Res 39:W347-W352. doi:10.1093/nar/gkr485

27. Roberts RJ, Vincze T, Posfai J, Macelis D (2015) REBASE-a database for DNA restriction and modification: enzymes, genes and genomes. Nucleic Acids Res 43:D298-D299. doi:10.1093 /nar/gkp874

28. King AMQ, Adams MJ, Carstens EB, Lefkowitz EJ (2012) Virus taxonomy. In: Virus taxonomy: ninth report of the international committee on taxonomy of viruses, London, Elsevier, pp 39-96

29. Marphy FA, Fauquet CM, Bishop DHL, Ghabrial SA, Jarvis AM, Martelli GP, Summers MD (1995) Virus taxonomy. In: Virus taxonomy: sixth report of the international committee on taxonomy of viruses, Wien, Springer, pp 1-60

30. Ackermann HW (2009) Phages classification and characterization. Methods Mol Biol 501:127-140. doi:10.1007/978-1-60327-1646_13

31. Bergh D, Borsheim KY, Bratbakand G, Heldal M (1989) High abundance of viruses found in aquatic environments. Nature 340(6233):467-468. doi:10.1038/340467a0

32. Hendrix R (2002) Bacteriophages: evolution of the majority. Theor Popul Biol 61:471-480. doi:10.1006/tpbi.2002.1590

33. Rohwer F (2003) Global phage diversity. Cell 113(2):141. doi:10.1016/S0092-8674(03)00276-9

34. Rauch HM, Pickett J (1961) Bordetella bronchiseptica bacteriophage. Can J Microbiol 7(2):125-133. doi:10.1139/m61-017

35. Mebel S, Rustenbach S, Lapaeva IA (1985) Isolierung von Bordetella-phagen aus bakterienstämen der impfstoffproduktion. Acta Biotechnol 5(4):329-332. doi:10.1002/abio.370050403

36. Mills S, Shanhan F, Stanton C, Hill C, Coffey A, Ross RP (2013) Movers and shakers: influence of bacteriophages in shaping mammalian gut microbiota. Gut Microbes 4(1):4-16. doi:10.4161 /gmic. 22371

37. Akerley BJ, Monack DM, Falkow S, Miller JF (1992) The bvgAS locus negatively controls motility and synthesis of flagella in Bordetella bronchiseptica. J Bacteriol 174(3):980-990

38. Irie Y, Matto S, Yuk MH (2004) The Bvg virulence control system regulates biofilm formation in Bordetella bronchiseptica. J Bacteriol 186(17):5692-5698. doi:10.1128/JB.186.17.56925698.2004

39. Vergara-Irigaray N (2005) Evaluation of the role of the Bvg intermediate phase in Bordetella pertussis during experimental respiratory infection. Infect Immun 73(2):748-760. doi:10.1128 /IAI.73.2.748-760.2005

40. Casjens SR, Gilcrease EB (2009) Determining DNA packaging strategy by analysis of termini of the chromosomes in tailed bacteriophage virions. Methods Mol Biol 502:91-111. doi:10.1007/9781-60327-565-1_7 